\title{
The effects of high dose interferon- $\beta 1$ a on plasma microparticles: Correlation with MRI parameters
}

\author{
Mary Lowery-Nordberg ${ }^{1,5}$, Erin Eaton ${ }^{1}$, Eduardo Gonzalez-Toledo ${ }^{3}$, Meghan K Harris ${ }^{4}$, Kathrine Chalamidas ${ }^{4}$,
} Jeanie McGee-Brown ${ }^{4}$, Chaitanya V Ganta ${ }^{2}$, Alireza Minagar ${ }^{4 *}$, David Cousineau ${ }^{1}$ and J Steven Alexander ${ }^{2,5}$

\begin{abstract}
Objectives: We previously reported a correlation between levels of microparticles carrying CD31 (PMP CD31+) and disease activity in MS. However, the effects of long term (12 month) treatment with high dose, high frequency interferon- $\beta$ 1a (RebifTM) on plasma levels of PMP ${ }^{C D 31+}$, PMP ${ }^{C D 146+}$, and PMP ${ }^{C D 54+}$ and MRI measures of disease activity have not yet been assessed.

Methods: During this prospective 1-year study, we used flow cytometry to measure changes in plasma microparticles (PMP) bearing CD31 (PMP ${ }^{\mathrm{CD} 31+}$ ), CD146 (PMP ${ }^{\mathrm{CD} 146+}$ ), and CD54/ICAM-1 (PMP ${ }^{\mathrm{CD} 54+}$ ) in 16 consecutive patients with relapsing-remitting MS (RRMS) before and after 3, 6, and 12 months of subcutaneous therapy with interferon-beta1a (44 micrograms, 3X weekly). At each visit, clinical exams and expanded disability status scale (EDSS) scores were recorded.

Results: Plasma levels of PMPCD31+, and PMPCD54+ were significantly reduced by treatment with IFN- $\beta 1$ a. PMPCD146 + appeared to decrease only at 3 months and did not persist at 6 and 12 months ( $p=0.0511$ ). In addition, the decrease in plasma levels of PMPCD31+ and PMPCD54+ levels at 12 months were associated with a significant decrease in the number and volume of contrast enhancing T1-weigthed lesions.

Conclusion: Our data suggest that serial measurement of plasma microparticles (PMP), particularly in the initial stages of MS (when neuro-inflammatory cascades are more intense), may serve as reliable and reproducible surrogate markers of response to IFN- $\beta$ la therapy for MS. In addition, the progressive decline in plasma levels of $\mathrm{PMP}^{\mathrm{CD} 31+}$ and PMP ${ }^{\mathrm{CD} 54+}$ further supports the concept that IFN- $\beta$ 1a exerts stabilizing effect on the cerebral endothelial cells during pathogenesis of MS.
\end{abstract}

\section{Introduction}

Multiple sclerosis (MS) is an immune-mediated neurodegenerative disease of the human central nervous system (CNS) characterized clinically by a relapsing-remitting course. Neuropathologically MS manifests with development of demyelinating lesions which affect both gray and white matter [1,2] disruption of the blood brain barrier (BBB) and transendothelial migration of lymphocytes and macrophages across inflamed CNS endothelial monolayers appear to be among the earliest $\mathrm{CNS}$ and spinal

\footnotetext{
* Correspondence: aminag@|suhsc.edu

${ }^{4}$ Department of Neurology, 1501 Kings Highway, Shreveport, LA 71130-3932, USA

Full list of author information is available at the end of the article
}

cord abnormalities in MS [3]. Activation of the cerebral endothelial cells and their binding with activated leukocytes are early crucial steps in creation of MS demyelinating lesions produced by immune cells $[4,5]$.

Cerebral endothelial cells, the main components of the $\mathrm{BBB}$, contain tight and adherens junctions which create a highly impenetrable and impermeable anatomic-physiologic barrier against circulating plasma neurotransmitters, cytokines, formed blood elements and soluble and insoluble molecular components of the circulating blood. Upon activation by pro-inflammatory cytokines such as IFN- $\gamma$ or TNF- $\alpha$, released by activated T-lymphocytes, endothelial cells shed small fragments of their membranes, known as endothelial microparticles (PMP)

\section{() Biomed Central}


[6]. These fragments contain some of the surface adhesion molecules and other endothelial markers of their parent cells [7], including, but not limited to, plateletendothelial cell adhesion molecule (PECAM-1/CD31), human endothelial marker CD146, and intercellular adhesion molecule (ICAM-1/CD54). Therefore while it is difficult to evaluate inflammatory endothelial markers in MS in situ, microparticles may provide a remote 'snapshot' of the surface of inflamed endothelium and provide information on the extent of platelet and leukocyte activity in MS.

We previously reported elevated plasma levels of PMP in MS patients and demonstrated that the plasma levels of $\mathrm{PMP}^{\mathrm{CD} 31+}$ may reflect acute endothelial injury with positive association with presence of contrast enhancing lesions [3]. Additionally, we reported that various sub-species of PMP form complex with different leukocytes and by formation of such complexes, they promote the inflammatory process by facilitating transendothelial migration of the leukocytes [6]. In the current study, we hypothesized that prospective serial measurement of three subspecies of PMP CD31 ${ }^{+}, \mathrm{PMP}^{\mathrm{CD} 146+}$, and PMP ${ }^{\mathrm{CD} 54+}$ may reflect disease activity and serve as a surrogate marker of therapeutic response to high dose, high frequency interferon- $\beta 1 \mathrm{a}$. We also assessed the correlation among these sub-species of PMP and MRI parameters.

\section{Methods}

During this one year prospective study, a cohort of 16 patients who met the Poser et al. criteria [8] for clinically definite MS, were studied. The clinical study was approved by Louisiana State University Health Sciences Center-institutional review board and all patients provided informed consent. For at least three months prior to initiation of treatment with IFN- $\beta 1 \mathrm{a}$, none of the subjects had received any immunosuppressive treatment or corticosteroids. All study subjects underwent neurological examination at the study entry prior to initiation of treatment with IFN- $\beta 1 \mathrm{a}$ and at 3,6 , and 12 months after initiation of treatment. At each visit, their expanded disability status scale (EDSS) scores were determined. Peripheral venous blood specimens (baseline) were collected prior to initiation and within the first 15 hours of treatment with IFN- $\beta 1 \mathrm{a}$ and at months 3,6 , and 12 after treatment initiation. The study subjects were treated with IFN- $\beta 1$ a 44 micrograms ug subcutaneously three times weekly. Because therapy cannot be withheld from MS patients, this study did not contain an arm consisting of untreated MS patients.

\section{Measurement of PMP using flow cytometry}

Using a 21 gauge needle, venous blood was obtained in citrate loaded vacutainer tubes. Measurements of plasma
PMP were performed within four hours from specimen collection. Briefly, blood specimen was centrifuged at $160 \times \mathrm{g}$ for 10 minutes to prepare platelet rich plasma (PRP). Next, the PRP specimen was centrifuged for 6 minutes at $1500 \times \mathrm{g}$ to generate platelet poor plasma (PPP). Then, each $25 \mu \mathrm{l}$ aliquot of PPP was incubated with $2 \mu \mathrm{l}$ of anti-CD31-PE, $2 \mu \mathrm{l}$ of anti-CD146, and $2 \mu \mathrm{l}$ of anti-CD54 at ambient temperature for 20 minutes with gentle shaking $(80 \mathrm{rpm})$. The PMPs in the sample were measured using a Beckman Coulter FC500 MCL flow cytometer system equipped with CXP software (Beckman Coulter, Miami, FL). The sample flow rate and particle detection settings are identical to that described previously [3]. Data collection is based on both multi-parameter and uni-parameter analyses of $\mathrm{PMP}^{\mathrm{CD} 31+}, \mathrm{PMPCD}^{146+}$, and PMP ${ }^{\mathrm{CD} 54+}$. To evaluate the possibility that leukocyte adhesion of microparticles might be measured, the anti-leukocyte common antigen CD45 was used in the cocktails. With the exception of whole blood analysis (used as a positive control) negligible $C D 45^{+}$events were detected in the PPP preparations.

\section{Statistical analysis}

Data were analyzed using Instat (Graphpad software, La Jolla CA) using repeated measures analysis of variance with Dunnett's post-testing to determine time points which differed statistically from starting baseline values. Data are shown as the average \pm standard error. Statistical differences in contrast enhancing T1-weighted lesion volumes at $\mathrm{t}=0$ were compared with $\mathrm{t}=12$ months using two tailed unpaired students t-test. Microparticle data are shown from $t=0$ to 12 months. PMP counts and volumes of contrast enhancing $\mathrm{T} 1$-weighted data are also plotted bi-directionally to show separate nonoverlapped groupings for PMP $\mathrm{PD}^{\mathrm{C} 4+}$ (ICAM-1) and $\mathrm{PMP}^{\mathrm{CD} 31+}$ with contrast enhancing T1weighted volumes.

\section{Magnetic resonance imaging protocol}

All study subjects underwent brain MRI by a $1.5 \mathrm{~T}$ machine with a standard head coil prior to initiation of therapy with IFN- $\beta 1 \mathrm{a}$ (Rebif). The neuroimaging protocol consisted of sagittal T1-weighted axial T1- and T2and proton density weighted images. All MR studies were done after infusion of gadolinium diethylenetriamine pentaacetic acid (Gd). We used post-contrast T1weighted and T2-weighted axial images for assessment of MS plaques. A neuroradiologist blinded to the subjects' clinical information, interpreted images and measured the volume and number of MS lesions. The second MRI of brain was obtained at month 12 after initiation of treatment with IFN- $\beta 1$ a. 


\section{Results}

Subjects with MS had a mean age of 31 years at study entry and had a mean duration of the illness of 14 months. There were 14 females and 2 male in this study. During the course of this study five subjects developed transient elevation of liver enzymes which eventually resolved. Apart from this, no other adverse events occurred as a result of IFN- $\beta 1$ a treatment. During this study we assessed the PMP profiles in MS patients pre- and post treatment with IFN- $\beta 1$ a (Rebif) and found that several but not all classes of plasma endothelial microparticles were altered in MS patients following treatment over 12 months. All data are reported as means \pm standard error.

\section{CD31}

Plasma levels of CD31 ${ }^{+}\left(\right.$PECAM- $\left.1^{+}\right)$microparticles/ul were reduced from a baseline level of $400 \pm 138 / \mathrm{ul}$ at $\mathrm{t}$ $=0$ to $167 \pm 68 /$ ul at 3 months, and $190 \pm 74 /$ ul at 6 months (not statistically significant). Data only reached statistical significance at 12 months when PMP ${ }^{\mathrm{CD} 31+}$ were $98 \pm 32 / \mathrm{ul}$ (Figure $1,^{*}=\mathrm{p}<0.05$ ). This may reflect individual variations in patient's responsiveness to treatment with IFN- $\beta 1$ a which did not become uniform enough to reach statistical significance until the $12^{\text {th }}$ month of treatment. However, comparison of plasma levels of $\mathrm{PMP}^{\mathrm{CD} 31+}$ at baseline versus one year of treatment with IFN- $\beta 1$ a indicates that this marker shows a significant difference in the quantity of shed PMP into plasma.

\section{CD146}

CD146 also known as melanoma cell adhesion molecule (Mel-CAM) or cell surface glycoprotein 'MUC18' [9]

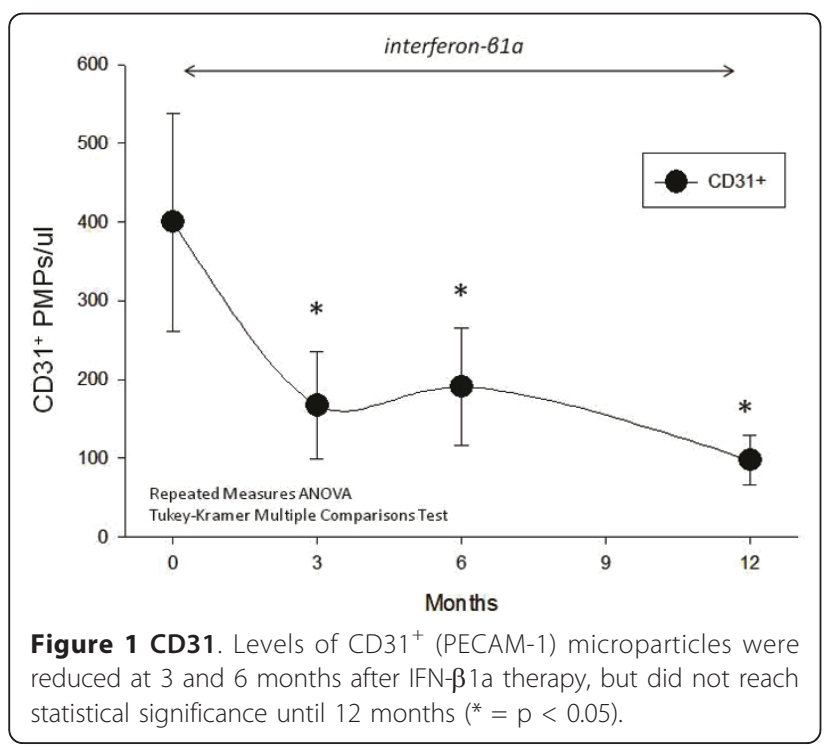

was marginally statistically altered by IFN $-\beta 1$ a treatment $(\mathrm{p}=0.0511)$ (Figure 2). After 3 months of IFN- $\beta 1$ a therapy $\mathrm{PMP}^{\mathrm{CD} 146+} / \mathrm{ul}$ were reduced from a baseline level $(\mathrm{t}=0)$ of $10.6 \pm 1.8 / \mathrm{ul}$, to $4.4 \pm 1.5 / \mathrm{ul}$ at 3 months; these levels returned to $7.9 \pm 1.6 /$ ul by 6 months, and $7.75 \pm 1.3 / \mathrm{ul}$ at 12 months.

\section{CD54}

By comparison CD54 $4^{+}\left(\mathrm{ICAM}-1^{+}\right)$plasma endothelial microparticles/ul were reduced from a baseline of $211 \pm$ $62.6 / \mathrm{ul}$ at $\mathrm{t}=0$, to $77.4 \pm 18.2 / \mathrm{ul}$ at 3 months $\left({ }^{*} \mathrm{p}<\right.$ $0.05)$, to $74.7 \pm 14.4 / \mathrm{ul}$ at 6 months $\left({ }^{*} \mathrm{p}<0.05\right)$ and $52.1 \pm 15.2$ at 12 months $(* \mathrm{p}<0.05)$ after IFN- $\beta 1 \mathrm{a}$ therapy compared to $t=0$ (Figure 3 ), suggesting that CD54 represents the most sensitive microparticle marker of disease activity.

We compared the plasma levels of $\mathrm{CD} 31^{+}$PMP with the volume of contrast enhancing T1-weighted lesions preand post treatment with IFN- $\beta 1$ a in order to detect any correlations between these two parameters. Figure 4 shows a bimodal graph correlating plasma levels of $\mathrm{PMP}^{\mathrm{CD} 31+}$ with the volumes of these lesions. We found that at 12 months both markers were significantly reduced by IFN- $\beta 1$ a therapy. Similarly Figure 5 also depicts the bimodal graph relationship between $\mathrm{PMP}^{\mathrm{CD} 54+}$ and volumes of contrast-enhancing T1-weighted lesions. Interestingly the magnitude of the reduction in these PMP profiles appears to be similar for both $\mathrm{PMP}^{\mathrm{CD} 54+}$ and $\mathrm{PMP}^{\mathrm{CD} 31+}$ when correlated with the volumes of contrastenhancing T1-weighted lesions. We did not find any significant correlation between the plasma levels of various classes of PMP and the volumes of T2-weighted lesions pre- and post treatment with IFN- $\beta 1 \mathrm{a}$.

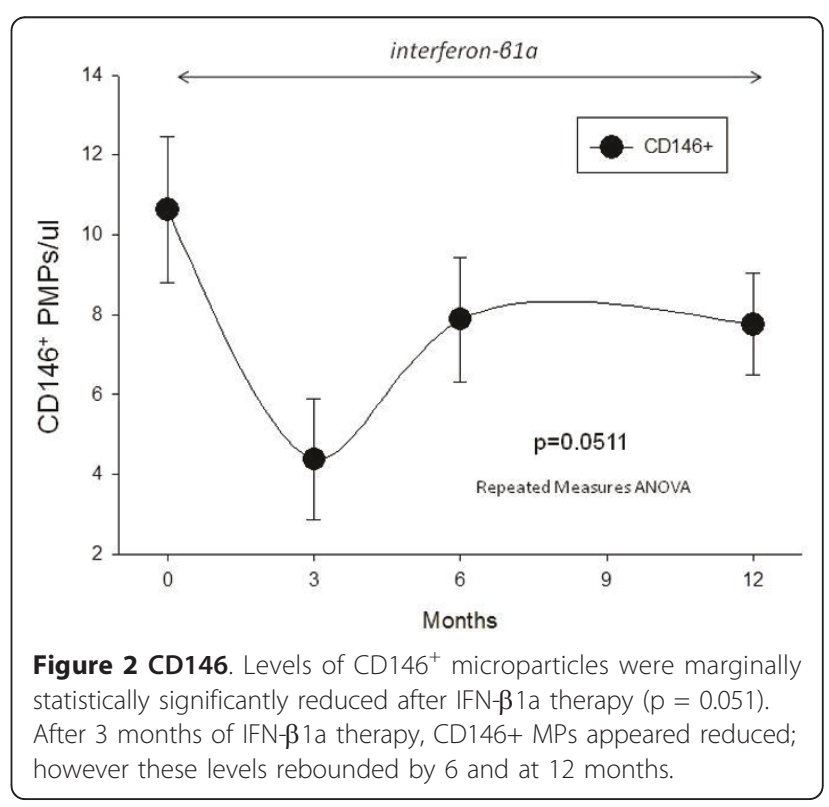




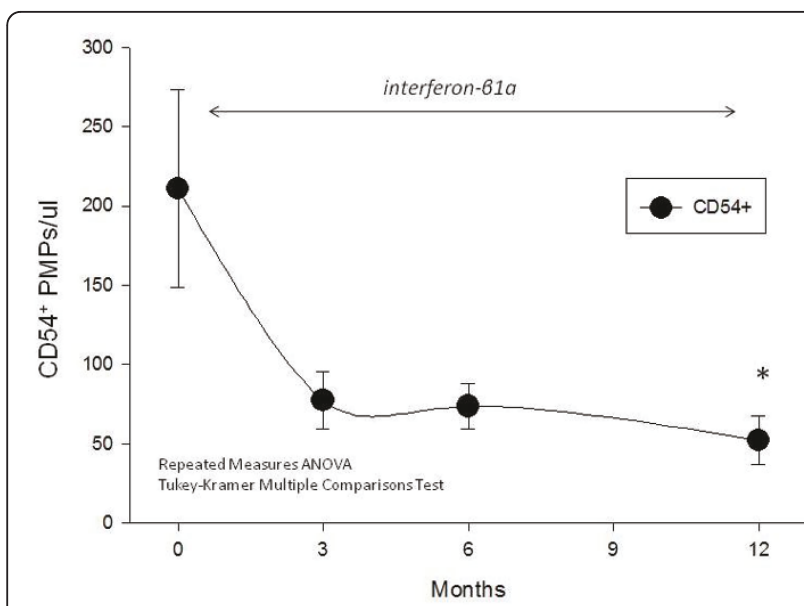

Figure 3 CD54. By comparison, CD54+ (ICAM-1+) plasma microparticles were statistically significantly reduced $(*, p<0.05)$ at 3,6 and 12 months after IFN- $\beta$ 1a therapy compared to $t=0$.

\section{Discussion}

High dose high frequency IFN- $\beta 1 \mathrm{a}$ is an FDA-approved treatment for MS which reduces annual relapse rate and improves brain MRI findings. The therapeutic benefits from IFN- $\beta 1$ a have been attributed to may involve effects on immune cells elevation of IL-10 and reduced synthesis of Th1 cytokines endothelial cell adhesion molecules MMPs resulting in lower leukocyte penetration of the CNS parenchyma, antigen presentation by microglia. We observed a decline in the plasma levels of $\mathrm{CD} 1^{+}$microparticles following treatment of MS patients with IFN- $\beta 1 \mathrm{a}$. The plasma levels of PMP ${ }^{\mathrm{CD} 31+}$ were found to be statistically reduced by IFN- $\beta 1$ a therapy at 3, 6 and 12 months. Because these studies were performed with single staining protocols we cannot

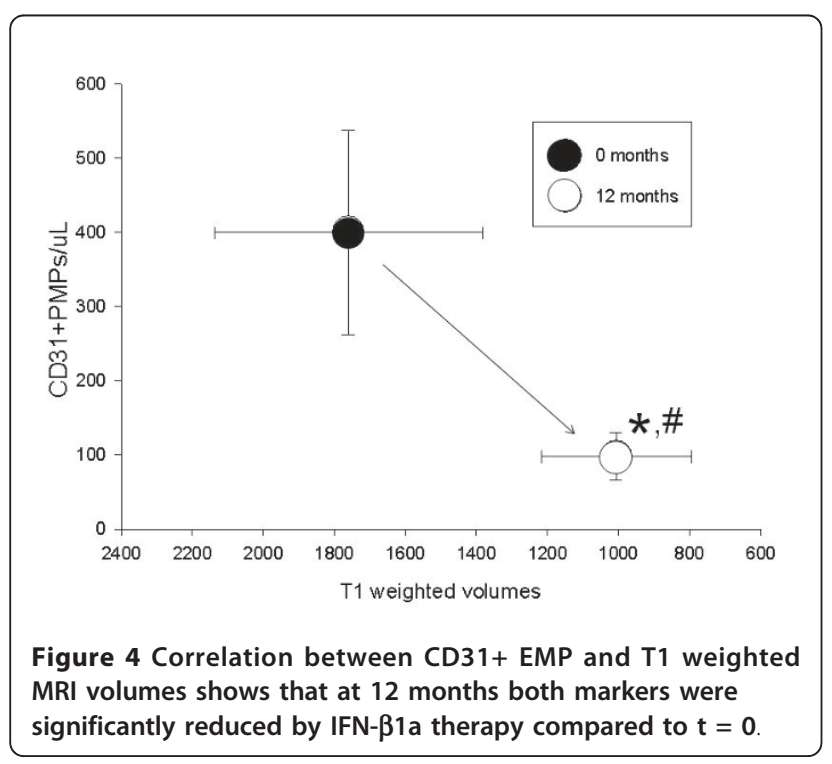

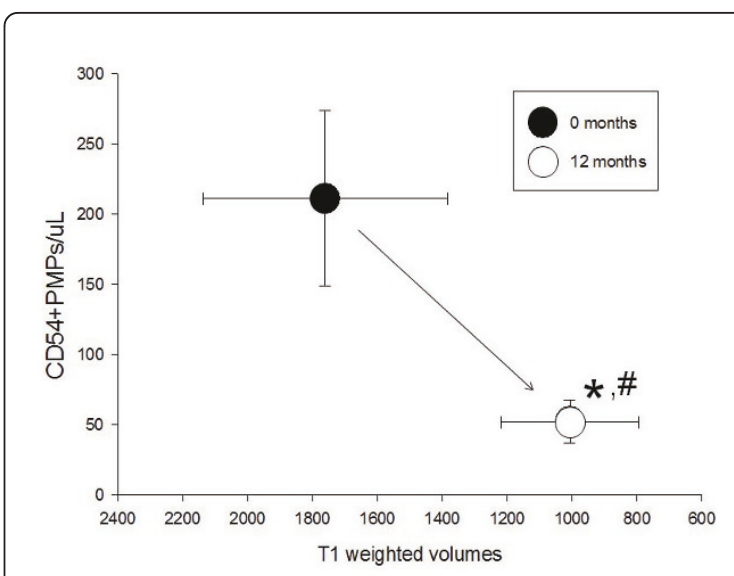

Figure 5 Correlation between $\mathrm{CD} 54^{+}$EMP and $\mathrm{T} 1$ weighted MRI volumes shows that at 12 months both markers were significantly reduced by IFN- $\beta 1$ a therapy compared to $t=0$.

specifically designate these particles as 'endothelial', and these must be considered to be a mixture of platelet and endothelial derived microparticles. CD31/PECAM-1 is known to mediate the transendothelial migration of leukocytes [10] in cytokine independent migration. Therefore, shedding of CD31 in microparticles, may represent an adaptive response aimed at limiting availability of CD31 on the endothelial, platelet or leukocyte membranes or alternatively may be another factor which facilitates transendothelial migration of the activated leukocytes. Therefore, a decrease in shedding of $\mathrm{PMP}^{\mathrm{CD} 31+}$ into plasma following treatment with IFN$\beta 1 \mathrm{~b}$ may indicate a lessened interaction between activated leukocytes and inflamed underlying endothelium, which in turn translates into stabilization of the blood brain barrier and decrease in the number of contrastenhancing T1-weighted lesions on brain MRI [3]; [11]. In addition, so-called 'insoluble' pools of CD31 found on microparticles may function as antagonists of leukocyte-bound CD31/PECAM-1 which functionally limits CD31 access, leading to reduced binding and transmigration.

Turning to another endothelial marker, PMP ${ }^{\mathrm{CD} 146+}$, we observed the effect of treatment of IFN- $\beta 1$ a on the plasma levels of this biological marker. CD146 is abundantly expressed on the surface of brain endothelial cells where it may act as a specific functional ligand for Th17 cells [12]. CD146 is also expressed on the surface of a subpopulation of activated T-cells [13] and mediates lymphocyte-endothelial adhesion. CD146+ microparticles were nearly statistically significantly reduced over 12 months of therapy with IFN- $\beta 1$ a. This change in $\mathrm{CD} 146^{+}$microparticle expression appeared at 3 months of therapy, and was not observed at 6 or 12 months. CD146, is also known as MUC18, which is 
considered to be a marker of endothelial cells, however it can also be found on a subset of T- and B-lymphocyte, NK cells [14], pericytes [15] and circulating endothelial cells [16]. Plasma levels of endothelial derived 'soluble' CD146 were inversely correlated with congestive heart failure [17], it is not clear whether how transient reductions in CD146 PMPs might be interpreted. Plasma levels of PMPCD146+ cannot yet predict a long term stabilizing effect of IFN- $\beta 1$ a on cerebral endothelial cells.

During the course of this prospective study, we also assessed the plasma levels of $\mathrm{PMP}^{\mathrm{CD} 54+}$ prior to treatment with IFN- $\beta 1 \mathrm{a}$ and at timed intervals (3, 6 and 12 months) after treatment. PMP ${ }^{\mathrm{CD} 54+}$ appears to mainly represent endothelial microparticles-derived ICAM-1 (CD54), but ICAM-1 can also be expressed by macrophages and T-cells. CD54 is constitutively expressed on the surface of endothelial cells [19], and its' expression is significantly upregulated by Th1 cytokines (TNF- $\alpha$, IL-1b, IFN- $\gamma$ ) which are known to be elevated during MS exacerbations [19]; [20]. While some studies have shown elevation of soluble forms of ICAM-1 (CD54) during active MS disease, [21] our study shows that levels of insoluble microparticles bearing CD54 in MS disease are significantly reduced by IFN- $\beta 1$ a therapy at 3, 6 and 12 months. In addition, previously Jy et al. [6] demonstrated that $\mathrm{PMP}^{\mathrm{CD} 54+}$ formed conjugates with monocytes and facilitated their migration through the endothelial cells monolayers and may represent an important mechanism supporting the penetration of immune cells into the CNS parenchyma in MS.

The correlation of plasma levels of $\mathrm{PMP}^{\mathrm{CD} 31+}$ and volumes of contrast-enhancing T1-weighted lesions on brain MRI is presented as a bimodal graph in Figure 4. We found that at 12 months both markers (PMP ${ }^{\mathrm{CD} 31+}$ and T1-weighted volumes) were significantly reduced by IFN- $\beta 1$ a therapy. Similarly, Figure 5 also depicts the bimodal graph relationship between $\mathrm{PMP}^{\mathrm{CD} 54+}$ and volumes of contrast-enhancing T1-weighted lesion on brain MR images. Interestingly, when plotted in this manner, the magnitude of the reduction in these profiles appears to be similar for both PMP ${ }^{\mathrm{CD} 31+}$ and $\mathrm{PMP}^{\mathrm{CD} 54+}$ with volumes of the contrast-enhancing $\mathrm{T} 1-$ weighted lesions. Whether and how these parameters are mechanistically linked to therapeutic benefit of IFNb1 $\alpha$ in MS is not clear but lower CD54 appears to reflect decreased endothelial activation. Because endothelial CD31/PECAM-1 is not as dramatically altered by exposure Th1 cytokines, as CD54 is for example [22], diminished CD31 levels suggest stabilization of membrane integrity and reduced platelet activation both of which could contribute to therapeutic benefit in MS.

\section{Conclusions}

Collectively, the results of our study lend support to the concept that administration of IFN- $\beta 1$ a to MS patients is associated with stabilization of the cerebral endothelial cells. This is reflected by concurrent decreases in the plasma levels of both $\mathrm{PMP}^{\mathrm{CD} 31+}$ and $\mathrm{PMP}^{\mathrm{CD} 54+}$ as well as volumes of contrast-enhancing T1-weighted lesions. We recommend that, at least during the early stages MS pathogenesis, measurement of certain sub-populations of plasma endothelial microparticles may serve as a reliable biological marker for disease activity and response to treatment with beta-interferons.

\section{Acknowledgements}

This study was supported by an educational grant from EMD Serono, Inc., Boston, MA. JSA and CVG were partly funded by a Feist Cardiovascular Imaging Award.

\section{Author details}

${ }^{1}$ Department of Pathology LSU Health Sciences Center-Shreveport, 1501 Kings Highway, Shreveport, LA 71130-3932, USA. ${ }^{2}$ Department of Molecular and Cellular Physiology, 1501 Kings Highway, Shreveport, LA 71130-3932, USA. ${ }^{3}$ Department of Radiology, 1501 Kings Highway, Shreveport, LA 71130 3932, USA. ${ }^{4}$ Department of Neurology, 1501 Kings Highway, Shreveport, LA 71130-3932, USA. ${ }^{5}$ Feist-Weiller Cancer Center, LSU Health Sciences CenterShreveport, 1501 Kings Highway, Shreveport, LA 71130-3932, USA.

\section{Authors' contributions}

MLN - Helped to conceive study and directed MP flow analysis work, EE coordinated patient sample collection and interacted with AM, EGT carried out all patient MRI analyses, MKH interviewed, consented and collected patient specimens, KC collected clinical patient information and coordinated between $\mathrm{AM}, \mathrm{MKH}$ and $\mathrm{EGT}$, JMB collected patient clinical information data and coordinated between AM, MKH and EGT, CVG interpreted data, statistics and helped write manuscript, AM helped conceive the study and helped to write manuscript, DC worked on MP flow cytometry and helped perform statistics, JSA helped to conceive study, interpret data, statistics, figures and helped to write manuscript. All authors read and approved the final manuscript.

\section{Competing interests}

The authors declare that they have no competing interests.

Received: 4 October 2010 Accepted: 9 May 2011 Published: 9 May 2011

\section{References}

1. Noseworthy JH, Lucchinetti C, Rodriguez M, Weinshenker BG: Multiple sclerosis. N Engl J Med 2000, 343:938-952.

2. Frohman EM, Racke MK, Raine CS: Multiple sclerosis-the plaque and its pathogenesis. N Engl J Med 2006, 354:942-955.

3. Minagar A, Jy W, Jimenez JJ, et al: Elevated plasma endothelial microparticles in multiple sclerosis. Neurology 2001, 56:1319-1324.

4. Brosnan CF, Battistini L, Gao YL, Raine CS, Aquino DA: Heat shock proteins and multiple sclerosis: a review. J Neuropathol Exp Neurol 1996, 55:389-402.

5. Springer TA: Traffic signals on endothelium for lymphocyte recirculation and leukocyte emigration. Annu Rev Physiol 1995, 57:827-872.

6. Jy W, Minagar A, Jimenez JJ, et al: Endothelial microparticles (EMP) bind and activate monocytes: elevated EMP-monocyte conjugates in multiple sclerosis. Front Biosci 2004, 9:3137-3144.

7. Horstman LL, Jy W, Minagar A, et al: Cell-derived microparticles and exosomes in neuroinflammatory disorders. Int Rev Neurobiol 2007, 79:227-268.

8. Poser CM, Paty DW, Scheinberg $L$, et al: New diagnostic criteria for multiple sclerosis: guidelines for research protocols. Ann Neurol 1983, 13:227-231. 
9. Kuske MD, Johnson JP: Assignment of the human melanoma cell adhesion molecule gene (MCAM) to chromosome 11 band q23.3 by radiation hybrid mapping. Cytogenet Cell Genet 1999, 87:258.

10. Muller WA, Weigl SA, Deng X, Phillips DM: PECAM-1 is required for transendothelial migration of leukocytes. J Exp Med 1993, 178:449-460.

11. Sheremata WA, Jy W, Delgado S, Minagar A, McLarty J, Ahn Y: Interferonbeta1a reduces plasma CD31+ endothelial microparticles (CD31+EMP) in multiple sclerosis. J Neuroinflammation 2006, 3:23.

12. Brucklacher-Waldert V, Stuerner K, Kolster M, Wolthausen J, Tolosa E: Phenotypical and functional characterization of T helper 17 cells in multiple sclerosis. Brain 2009, 132:3329-3341.

13. Guezguez B, Vigneron P, Lamerant N, Kieda C, Jaffredo T, Dunon D: Dual role of melanoma cell adhesion molecule (MCAM)/CD146 in lymphocyte endothelium interaction: MCAM/CD146 promotes rolling via microvilli induction in lymphocyte and is an endothelial adhesion receptor. J Immunol 2007, 179:6673-6685.

14. Elshal MF, Khan SS, Takahashi Y, Solomon MA, McCoy JP Jr: CD146 (Mel(AM), an adhesion marker of endothelial cells, is a novel marker of lymphocyte subset activation in normal peripheral blood. Blood 2005, 106:2923-2924.

15. Maier CL, Shepherd BR, Yi T, Pober JS: Explant outgrowth, propagation and characterization of human pericytes. Microcirculation 2010, 17:367-380.

16. Langer HF, von der Ruhr JW, Daub K, et al: Capture of endothelial progenitor cells by a bispecific protein/monoclonal antibody molecule induces reendothelialization of vascular lesions. J Mol Med 2010, 88:687-699.

17. Barisione C, Garibaldi S, Ghigliotti G, et al: CD14CD16 monocyte subset levels in heart failure patients. Dis Markers 2010, 28:115-124.

18. Lawson C, Wolf S: ICAM-1 signaling in endothelial cells. Pharmacol Rep 2009, 61:22-32.

19. Navikas V, He B, Link J, et al: Augmented expression of tumour necrosis factor-alpha and lymphotoxin in mononuclear cells in multiple sclerosis and optic neuritis. Brain 1996, 119(Pt 1):213-223.

20. Hohnoki K, Inoue A, Koh CS: Elevated serum levels of IFN-gamma, IL-4 and TNF-alpha/unelevated serum levels of IL-10 in patients with demyelinating diseases during the acute stage. J Neuroimmunol 1998, 87:27-32.

21. Hartung HP, Michels M, Reiners K, Seeldrayers P, Archelos JJ, Toyka KV Soluble ICAM-1 serum levels in multiple sclerosis and viral encephalitis. Neurology 1993, 43:2331-2335.

22. Henninger DD, Panes J, Eppihimer M, et al: Cytokine-induced VCAM-1 and ICAM-1 expression in different organs of the mouse. J Immunol 1997 158:1825-1832

doi:10.1186/1742-2094-8-43

Cite this article as: Lowery-Nordberg et al:: The effects of high dose interferon- $\beta 1 \mathrm{a}$ on plasma microparticles: Correlation with MRI parameters. Journal of Neuroinflammation 2011 8:43.

\section{Submit your next manuscript to BioMed Central and take full advantage of:}

- Convenient online submission

- Thorough peer review

- No space constraints or color figure charges

- Immediate publication on acceptance

- Inclusion in PubMed, CAS, Scopus and Google Scholar

- Research which is freely available for redistribution

Submit your manuscript at www.biomedcentral.com/submit
Ciomed Central 